\title{
Transcranial Magnetic Stimulation and Diffusion Tensor Tractography for Evaluating Ambulation after Stroke
}

\author{
Bo-Ram Kim, ${ }^{\text {a }}$ Won-Jin Moon, ${ }^{\mathrm{b}}$ Hyuntae Kim, ${ }^{\mathrm{a}}$ Eunhwa Jung, ${ }^{\mathrm{c}}$ Jongmin Lee ${ }^{\mathrm{a}}$ \\ ${ }^{a}$ Department of Rehabilitation Medicine, Konkuk University School of Medicine and Konkuk University Medical Center, Seoul, Korea \\ ${ }^{b}$ Department of Radiology, Konkuk University School of Medicine and Konkuk University Medical Center, Seoul, Korea \\ 'Department of Rehabilitation Medicine, The Graduate School of Konkuk University and Konkuk University Medical Center, Seoul, Korea
}

Background and Purpose We aimed to investigate the usefulness of combining transcranial magnetic stimulation (TMS) and diffusion tensor tractography (DTT) to evaluate corticospinal tract (CST) integrity and subsequently predict ambulatory function after middle cerebral artery (MCA) stroke.

Methods Forty-three patients with first MCA stroke underwent TMS and DTT to evaluate CST integrity. Patients were classified into four groups according to the presence of motor-evoked potentials (MEPs) obtained from the tibialis anterior muscle and CST integrity. Motor impairment and functional status were assessed using the Fugl-Meyer Assessment, Functional Ambulation Category, and Korean modified Barthel Index, both at the time of admission and after 4 weeks of rehabilitation.

Results Patients with the presence of both measurable MEPs and a preserved CST showed better motor recovery and ambulatory function than other groups at the 4-week follow-up. Intact CSTs were not visualized in patients without detectable MEPs. Among the patients displaying MEPs, those with preserved CSTs showed better recovery of paretic lower extremities.

Conclusions Combined assessment using TMS and DTT to evaluate CST integrity confers advantages in predicting motor and ambulation recovery in patients with MCA stroke.

\author{
Correspondence: Jongmin Lee \\ Department of Rehabilitation Medicine, \\ Konkuk University Medical Center, \\ 120-1 Neungdong-ro, Gwangjin-gu, \\ Seoul 05030, Korea \\ Tel: $+82-2-2030-5345$ \\ Fax: +82-2-2030-5379 \\ E-mail: leej@kuh.ac.kr \\ Received: December 16, 2015 \\ Revised: April 25, 2016 \\ Accepted: April 27, 2016 \\ The authors have no financial conflicts of \\ interest.
}

Keywords Stroke; Locomotion; Corticospinal tract; Rehabilitation

\section{Introduction}

Predicting the level of functional recovery expected in patients with post-stroke hemiplegia is highly important when making an overall treatment plan. Rehabilitation therapies may be more effective if they are tailored to the intact anatomical and physiological structures of each individual patient. Thus, many clinicians have made an effort to precisely and objectively evaluate post-stroke brain injury and its prognosis. The corticospinal tract (CST) is the primary motor pathway in the central nervous system, and many studies indicate that CST preservation is essential for good recovery of impaired motor function in patients following stroke. ${ }^{1,2}$ Motor-evoked potentials (MEPs), which can be obtained by transcranial magnetic stimulation (TMS) of the motor cortex, are used to study various central nervous system disorders. ${ }^{3}$ The absence or low amplitude of TMS responses suggests loss of neurons or axons in the CST, which can be used as an early stage prognostic indicator of motor and functional recovery in patients with stroke. ${ }^{4-7}$ Diffusion tensor imaging (DTI) is a method for visualizing anisotropic water molecule diffusion characteristics in the white matter of the brain. ${ }^{8}$ More specifically, DTI visualizes the diffusion of water molecules along axo- 
nal tracts within high-density white matter nerve fibers. ${ }^{9}$ Diffusion tensor tractography (DTT) is a method that produces a three-dimensional representation of CST structure (or integrity), which is derived from DTI. Several studies have demonstrated that DTT is useful for predicting motor outcome in patients with stroke. ${ }^{10-12}$ An increase in ipsilateral connectivity has a positive correlation with slower walking speeds, higher asymmetrical fractional anisotropy values, and is associated with greater walking impairments in DTI analysis. ${ }^{13}$ Alterations in the balance of corticomotor excitability in lower limbs at the chronic stage of stroke are related to poor motor recovery after stroke. ${ }^{14}$ Tibialis anterior muscle MEPs registered in the subacute phase of stroke may provide important prognostic information for lower extremity motor recovery ${ }^{15}$ and ambulatory function. ${ }^{16}$

Although several recent studies have compared the usefulness of TMS and DT in predicting upper extremity motor outcome in patients with stroke, ${ }^{17,18}$ there have been limited studies combining both MEP and CST data to predict lower extremity motor and ambulatory function. In the present study, we examined the usefulness of combining TMS and DTT to evaluate CST integrity and subsequently predict ambulatory and lower extremity functional recovery after middle cerebral artery (MCA) stroke.

\section{Methods}

\section{Subjects}

Patients with first stroke episodes who were admitted for rehabilitation to Konkuk University Medical Center from November 2010 to December 2013 were recruited. Only those with ischemic or hemorrhagic strokes in the MCA regions were included. TMS and DTI were performed at the same time, 3-8 weeks after stroke onset. The mean age of patients was $62.8 \pm 12.3$ years, and the average time between stroke onset and combined TMS and DTI procedures was $38.8 \pm 15.8$ days. All patients underwent comprehensive rehabilitation treatment for 4 weeks.

Patient exclusion criteria for this study included the following: (1) previous history of a stroke; (2) inability to walk before stroke onset; (3) medication use, such as sedatives, anticonvulsants, or muscle relaxants; (4) a diagnosis of diabetes or other peripheral neuropathy; (5) low-level consciousness; (6) contraindication to magnetic resonance imaging; or (7) refusal of DTI.

The Fugl-Meyer Assessment (FMA), ${ }^{19}$ Functional Ambulation Category (FAC), ${ }^{20}$ and Korean modified Barthel Index (K-MBI) ${ }^{21}$ were used to assess motor function, walking ability, and functional status in all patients at the time of admission and after 4 weeks of rehabilitation. The FMA is an index for evaluating motor function and is comprised of five domains: joint movement and pain, sensation, balance, upper limb motor function, and lower limb motor function. ${ }^{19}$ These five domains are further divided into subcategories, each of which is graded on a threepoint scale: 0 , complete inability to perform the task; 1 , partial ability to perform the task; and 2, perfect ability to complete the task. The FAC is based on characterizing the level of assistance required during a $15 \mathrm{~m}$ walk. The six FAC scores are as follows: 0 , the patient cannot walk at all or requires physical assistance from two persons; 1, a need for continuous manual contact of one person to support body weight; 2, a need for intermittent light touch from one person to assist balance; 3, a need for verbal cuing or ambulation on level surfaces without manual contact; 4, independent on level surfaces; 5 , can walk independently anywhere. We divided the patients into three groups according to the FAC scores; Nonfunctional ambulation (FAC score: 0), Dependent ambulation (FAC score: 1-3), Independent ambulation (FAC score: 4-5). ${ }^{20,22}$ After 4 weeks of rehabilitation treatment, evaluation of clinical scales (FMA, FAC, K-MBI) was carried out in all patients.

\section{Transcranial magnetic stimulation}

Cerebral TMS was performed using a Magstim 200 stimulator (The Magstim Company Limited, Wales, UK) with a $9 \mathrm{~cm}$ mean diameter circular coil, while patients remained comfortably seated. Cortical stimulation was performed by with the coil held tangentially over the vertex. MEPs were obtained from both tibialis anterior muscles in a relaxed state. The resting motor threshold was defined (according to the international standard) as follows: the minimum stimulation strength required to produce an amplitude of $50 \mu \mathrm{V}$ or more for at least 5 out of 10 consecutive TMS attempts. ${ }^{23}$ MEPs were measured using 120\% of the resting motor threshold stimulation strength. Each site was stimulated 5 times at 10 -second minimum intervals, from which the shortest latency and the largest peak-to-peak amplitudes were adopted.

\section{DTI data acquisition and analysis}

All imaging was performed on a 3.0 Tesla magnetic resonance imaging system (Signa HDxt, GE Medical Systems, Milwaukee, Wisconsin, USA) with a standard eight-channel phase array head coil. For each of the 15 noncollinear and noncoplanar diffusionsensitizing gradients, approximately 70 contiguous slices, parallel to the anterior commissure-posterior commissure line, were acquired. Imaging parameters were defined as follows: matrix = $120 \times 120$ matrix, field of view $=240 \times 240 \mathrm{~mm}^{2}$, TE (echo time) $=84 \mathrm{~ms}$, TR (repetition time) $=16,000 \mathrm{~ms}, \mathrm{~b}=800 \mathrm{~mm}^{2} \mathrm{~s}^{-1}$, and slice thickness $=2 \mathrm{~mm}$. In addition to DTI, conventional T2 fluid-attenuated inversion recovery images were obtained.

Raw image data were transferred in a digital imaging and communication in medicine format. All DTI images were correct- 


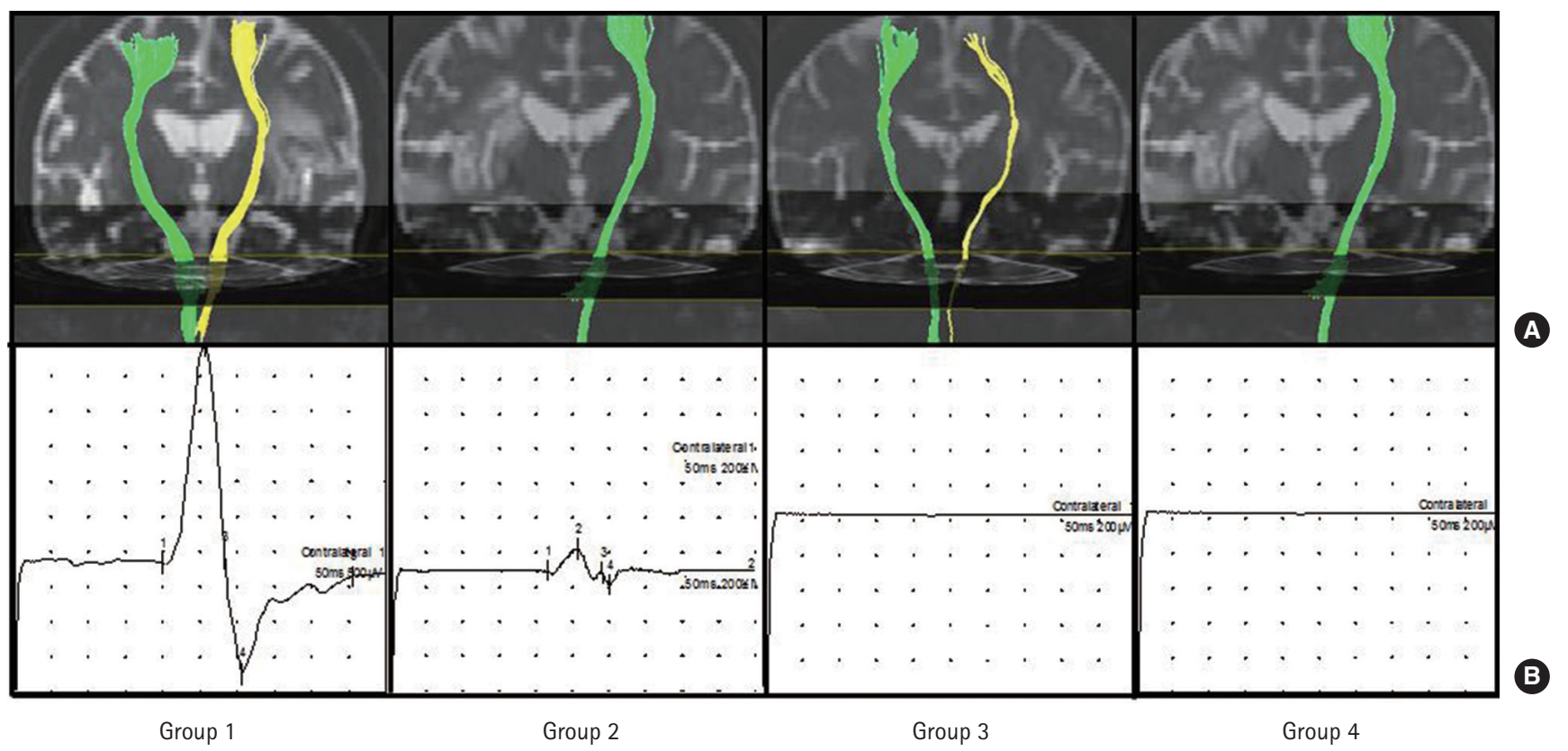

Figure 1. Classification of subjects according to DTT and TMS results. (A) Coronal DTT images. (B) TMS results. Groups are classified as follows: Group 1, DTT(+)/MEP(+) (preserved CST and presence of MEPs); Group 2, DTT(-)/MEP(+) (absence of CST and presence of MEPs); Group 3, DTT(+)/MEP(-) (preserved CST and absence of MEPs); and Group 4, DTT(-)/MEP(-) (absence of CST and MEPs). DTT, diffusion tensor tractography; TMS, transcranial magnetic stimulation; MEP, motor-evoked potential; CST, corticospinal tract.

ed for eddy-current-induced image distortions using FSL software (The Image Analysis Group, FMRIB, Oxford, UK, http://www. fmrib.ox.ac.uk/fsl). DTI analyses were performed by an experienced rehabilitation medicine doctor using DTI studio software (www.mristudio.org, Johns Hopkins Medical Institute, Baltimore, $\mathrm{MD}$ ). All parameters were measured twice, and the results were averaged. To reconstruct the CST, the first region of interest was freehand-drawn in the upper pons, through which motor fibers descend, and the second region of interest was drawn in the basis pontis of the lower pons, using the "AND" operation. Tract fibers passing through both regions of interest were designated as final tracts of interest. ${ }^{18,24}$

Patients were divided into four groups according to the presence of MEPs in affected tibialis anterior muscles and observed CST integrity, as measured by DTI. Groups were defined according to the following classifications: Group 1, DT(+) and MEP(+); Group 2, DT(-) and MEP(+); Group 3, DTT(+) and MEP(-); Group 4, DTT(-) and MEP(-) (Figure 1).

Lesion volumes were calculated by adding the individual section lesion areas multiplied by section separation using the MRIcro software package. ${ }^{25}$

\section{Statistical analysis}

The Kruskal-Wallis test, a non-parametric signed rank test, was used ${ }^{26}$ to analyze motor impairment and functional status between groups. Analysis of covariance was performed to deter- mine the prognostic factor of ambulatory function after 4 weeks. The initial FMA score was used as the covariate in order to adjust the motor impairment and initial lesion volume at the time of admission. Statistical significance was determined at $P<0.05$. Data analyses were performed using SPSS version 17.0 software (SPSS Inc., Chicago, IL, USA).

\section{Results}

Forty-three patients ( 23 males and 20 females, with a mean age of $62.8 \pm 12.3$ years) were recruited, with $24,9,0$, and 10 patients in Groups 1, 2, 3, and 4, respectively (Figure 1). There were no differences in age, sex, lesion side, or number of days from onset between any of the groups. However, a higher hemorrhage rate and increase lesion volume were observed in Group 4 (Table 1).

CSTs were completely disrupted in all patients without detectable MEPs. Thus, no patients were included in Group 3. Patients with the presence of both undisrupted CSTs and detectable MEPs (Group 1) showed higher FAC, FMA (of lower extremities), and K$\mathrm{MBI}$ scores than other groups at all time points $(P<0.05$, compared with all groups). Among the patients with detected MEPs (Groups 1 and 2), patients with preserved CSTs showed better motor function of paretic lower extremities and ambulatory function $(P<0.05$; Table 2). There were significant differences in FAC and $\mathrm{K}-\mathrm{MBI}$ scores at the 4-week follow-up between groups 
Table 1. Profiles of study populations

\begin{tabular}{|c|c|c|c|c|}
\hline Group & $\begin{array}{c}\text { Group } 1 \\
\text { DTT(+)/MEP(+) } \\
(n=24)\end{array}$ & $\begin{array}{c}\text { Group } 2 \\
\text { DTT(-)/MEP(+) } \\
(n=9)\end{array}$ & $\begin{array}{c}\text { Group } 3 \\
\text { DTT(+)/MEP(-) } \\
(n=0)\end{array}$ & $\begin{array}{c}\text { Group } 4 \\
\text { DTT(-)/MEP(-) } \\
(n=10)\end{array}$ \\
\hline Age (year) & $63.0 \pm 12.8$ & $67.3 \pm 7.6$ & & $58.3 \pm 13.7$ \\
\hline Female:male ratio & $11: 13$ & $4: 5$ & & $5: 5$ \\
\hline Onset to DTI/TMS (day) & $39.5 \pm 17.9$ & $28.9 \pm 9.7$ & & $45.9 \pm 10.6$ \\
\hline Lesion side (right:left) & $16: 8$ & $3: 6$ & & $5: 5$ \\
\hline Stroke type (infarction:hemorrhage) ${ }^{*}$ & $22: 2$ & $9: 0$ & & $5: 5$ \\
\hline \multicolumn{5}{|l|}{ Lesion location } \\
\hline Cortex & $3(12.5)$ & 0 & & 0 \\
\hline Subcortex & $13(54.2)$ & $5(55.6)$ & & $7(70.0)$ \\
\hline Cortex+Subcortex & 8 (33.3) & $4(44.4)$ & & $3(30.0)$ \\
\hline Lesion volume $\left(\mathrm{mm}^{3}\right)^{*}$ & $3,633 \pm 4,458.7$ & $3,498.9 \pm 4,659.6$ & & $7,562.6 \pm 3,213.5$ \\
\hline
\end{tabular}

Values indicate number of patients or means \pm standard deviation.

DT, diffusion-tensor tractography; MEP, motor-evoked potentials.

${ }^{*} P$ value $<0.05$.

Table 2. Comparison of functional outcomes among groups

\begin{tabular}{llccc}
\hline Group & & DTT(+)/MEP(+) & DTT(-)/MEP(+) & DTT(-)/MEP(-) \\
\hline FAC score & Initial $^{*}$ & $1.7 \pm 1.7$ & $0.2 \pm 0.4$ & $0.2 \pm 0.6$ \\
FMA_LE score & After four weeks* $^{*}$ & $2.9 \pm 1.7$ & $1.6 \pm 1.1$ & $0.8 \pm 1.1$ \\
K-MBI score & Initial* $^{*}$ & $23.0 \pm 11.1$ & $6.8 \pm 8.0$ & $7.0 \pm 4.1$ \\
& After four weeks* $^{*}$ & $26.0 \pm 9.5$ & $9.6 \pm 7.9$ & $9.5 \pm 5.7$ \\
\hline
\end{tabular}

Values indicate means \pm standard deviation.

FAC, Functional Ambulation Category; K-MBI, Korean modified Barthel Index; FMA_LE, Fugl-Meyer Assessment lower extremity score for the paretic side.

${ }^{*} P$ value $<0.05$.

Table 3. Lesion volume and initial FMA score of lower limb adjusted means ( \pm standard error) of FAC \& K-MBI scores at the 4-week follow-up according to TMS and DT results

\begin{tabular}{lcccc}
\hline & DT(+)/MEP(+) & DTT(-)/MEP(+) & DTT(-)/MEP(-) & $P$ value \\
\hline FAC at 4 weeks later & $2.8 \pm 0.3$ & $1.8 \pm 0.5$ & $0.7 \pm 0.5$ & $<0.05$ \\
MBI at 4 weeks later & $60.3 \pm 5.1$ & $32.8 \pm 8.0$ & $34.2 \pm 8.8$ & $<0.05$ \\
\hline
\end{tabular}

TMS, transcranial magnetic stimulation; FMA, Fugl Meyer Assessment; FAC, Functional Ambulation Category; FMA_LE, Fugl Meyer Assessment of lower extremity; MBI, Modified Barthel Index.

Table 4. Ambulation status at discharge according to TMS and DTT results

\begin{tabular}{|c|c|c|c|c|}
\hline FAC level & DTT(+)/MEP(+) & DTT(-)/MEP(+) & DTT(-)/MEP(-) & $P$ value \\
\hline Nonfunctional ambulation & $4(16.66)$ & $2(22.22)$ & $6(60.0)$ & $<0.05$ \\
\hline Dependent ambulation & $9(37.5)$ & 7 (77.77) & $4(40.0)$ & \\
\hline Independent ambulation & $11(45.83)$ & 0 & 0 & \\
\hline
\end{tabular}

Values represent numbers of patients (\%). Nonfunctional ambulation, FAC score: 0; Dependent ambulation, FAC score: 1-3; Independent ambulation, FAC score: 4-5.

FAC, Functional Ambulation Category.

after incorporating the adjusted lesion volume and initial FMA score of lower extremities (Table 3).

In Group 1, DTT(+) and MEP(+), 16.66\% of patients had a nonfunctional ambulation status at admission, and $45.83 \%$ of patients achieved independent ambulation at discharge. However, in Group 4, DTT(-) and MEP(-), 60\% of patients had a nonfunctional ambulation status at admission, and no patients achieved independent ambulation at discharge (Table 4).

\section{Discussion}

In the current study, we investigated whether TMS and DTT measures of CST status during the subacute stage of MCA stroke can predict lower extremity motor outcomes and ambulatory 
function at discharge. We found that outcomes for lower extremity and ambulatory function among patients exhibiting both MEPs and preserved CSTs were superior to the patients who did not exhibit preservation of adjusted lesion volume and initial motor impairment of lower extremities, even though all patients participated in a 4 week rehabilitation program.

In the first 3 months after stroke, various degrees of motor recovery are observed, with the most significant recovery occurring within the first 4 weeks. ${ }^{27}$ The severity of muscle weakness at the time of stroke is considered a poor prognostic indicator compared to other factors, such as history of previous stroke, sphincter incontinence, cognitive function, or impaired functional status during hospitalization. ${ }^{28}$ It has been reported that good recovery rates were as rare as $15 \%-27 \%$ in patients with severe, post-stroke upper extremity paralysis. ${ }^{28-30}$ The present study found that initial motor impairment influenced ambulation function 4 weeks after stroke, and that results from TMS and DT were useful in predicting ambulation recovery even after being adjusted for initial motor impairment.

Clinicians use various assessment tools to evaluate brain damage in patients with stroke, including clinical rating scales, diagnostic radiology, or neurophysiological tests. In general, clinical rating scales for stroke are divided into two assessment methods: evaluation of brain damage in patients with acute stroke, ${ }_{1}^{31}$ and measurement of brain damage induced motor impairment in patients with subacute stroke. ${ }^{32}$ There are several diagnostic radiology methods used to assess brain damage, these include: (1) computed tomography or magnetic resonance imaging to detect the location and size of the lesion, ${ }^{33}$ (2) single-photon emission computed tomography or positron emission tomography scans to evaluate brain metabolism, ${ }^{34}$ (3) DTI to evaluate white matter tract integrity, ${ }^{10-12}$ and (4) measuring MEPs to monitor changes in motor system function. ${ }^{3,23}$

MEPs induced by TMS of the motor cortex is a widely used method to investigate central nervous system disorders. The absence of low amplitude MEPs in patients with stroke suggests loss of motor neurons or axons. ${ }^{6}$ Thus, this method is used to predict patient prognosis in the early post-stroke period. Several studies have focused on using TMS to predict prognosis of motor recovery. Among patients with acute ischemic stroke, those with measurable MEPs showed better motor and functional recovery than those without MEPs. Moreover, among the patients with measurable MEPs, those who had delayed central conduction time showed poor recovery. Thus, measurement of MEPs could be an early prognostic indicator to evaluate motor function and functional recovery. ${ }^{4,16,35}$ Furthermore, it was reported that MEP measurement was also useful in predicting prognoses during various follow-up periods between 1 and 6 months post-stroke. ${ }^{16}$
DTT is a radiological analysis used to evaluate CST integrity and TMS efficacy. In various studies evaluating prognosis of stroke recovery using DTT, upper extremity functional outcomes can be predicted through differentiation of distinct CST integrity types among patients with lacunar infarction and thalamic hemorrhage. ${ }^{36,37}$ When we compared post-stroke walking impairment to CST integrity, patients with relatively greater ipsilateral connectivity between the contralesional motor cortex and paretic lower limb were more behaviorally impaired, and more structural damage was present in the ipsilesional hemisphere CST. ${ }^{13}$

Recently, many studies have attempted to understand the correlation between different rating scales and how to more accurately predict prognoses after stroke. ${ }^{38-40}$ In a CST-analysis study using TMS and DT, Kwon et al. ${ }^{17}$ measured affected-side, upper extremity MEPs; using this method, TMS showed higher positive predictability, whereas DT showed higher negative predictability for determining upper extremity motor outcomes in patients with corona radiata infarction. Outcomes were also similar to those of patients with intracerebral hemorrhage. ${ }^{18}$

Previous studies have reported recovery outcomes for both upper extremity and ambulatory function based on upper extremity MEP measurements and CST integrity evaluated by DT: ${ }^{7}$ however, in these studies, lower extremity, rather than upper extremity, MEPs were used to evaluate ambulatory function. Researchers have hypothesized that the predictive power of lower extremity evaluations would be higher than that of upper extremities. Thus, in the present study, we evaluated the correlation between MEPs and CST integrity, as well as that of lower extremity and ambulatory function. We found that the prognosis of both lower extremity and ambulatory function among patients with detectable lower extremity MEPs was superior to that of patients without detectable MEPs. Moreover, prognosis of patients presenting with both MEPs and preserved CSTs was superior to that of patients presenting with only MEPs and impaired CSTs.

However, this study has several limitations, which stem mostly from its small sample size, making it difficult to predict overall outcome in stroke patients. Moreover, there is a limitation in predicting ambulatory function at a particular time point due to the inconsistency in timing of TMS and DT, ranging from 3-8 weeks after stroke onset. In the future, we plan to replicate this study with a larger sample size and a longer follow-up period in a more controlled study design.

\section{Conclusions}

Among the methods used in this study, DTT was highly useful in predicting detailed prognoses among stroke patients with de- 
tectable MEPs. TMS was also useful in predicting detailed prognoses among stroke patients with completely disrupted CSTs. In conclusion, the combined evaluation of CST integrity by TMS and DTT offers an advantage in predicting motor and ambulation recovery in patients with MCA stroke.

\section{References}

1. Hallett M, Wassermann EM, Cohen LG, Chmielowska J, Gerloff C. Cortical mechanisms of recovery of function after stroke. NeuroRehabilitaion 1998;10:131-142.

2. Watanabe T, Honda Y, Fujii Y, Koyama M, Matsuzawa H, Tanaka R. Three-dimensional anisotropy contrast magnetic resonance axonography to predict the prognosis for motor function in patients suffering from stroke. J Neurosurg 2001;94: 955-960.

3. Trompetto C, Assini A, Buccolieri A, Marchese R, Abbruzzese G. Motor recovery following stroke: a transcranial magnetic stimulation study. Clin Neurophysiol 2000;111:1860-1867.

4. Escudero JV, Sancho J, Bautista D, Escudero M, López-Trigo J. Prognostic value of motor evoked potential obtained by transcranial magnetic brain stimulation in motor function recovery in patients with acute ischemic stroke. Stroke 1998;29:18541859.

5. Kim GW, Won YH, Park SH, Seo JH, Ko MH. Can motor evoked potentials be an objective parameter to assess extremity function at the acute or subacute stroke stage? Ann Rehabil Med 2015;39:253-261.

6. Currà $A$, Modugno N, Inghilleri $M$, Manfredi $M$, Hallett $M$, Berardelli A. Transcranial magnetic stimulation techniques in clinical investigation. Neurology 2002;59:1851-1859.

7. Wu G, Wang L, Liu J, Mao Y, Qin G. Minimally invasive procedures reduced the damages to motor function in patients with thalamic hematoma: observed by motor evoked potential and diffusion tensor imaging. J Stroke Cerebrovasc Dis 2013;22: 232-240.

8. Neil J, Miller J, Mukherjee P, Hüppi PS. Diffusion tensor imaging of normal and injured developing human brain - a technical review. NMR Biomed 2002;15:543-552.

9. Mori S, Wakana S, Nagae-Poetscher LM, van Ziji PCM. MRI atlas of human white matter. 1st ed. Italy: Elsevier, 2005.

10. Jang SH, Bai D, Son SM, Lee J, Kim DS, Sakong J, et al. Motor outcome prediction using diffusion tensor tractography in pontine infarct. Ann Neurol 2008;64:460-465.

11. Yang DS, Kim DS, Kim YH, Jang SH. Demonstration of recovery of a severely damaged corticospinal tract: a diffusion tensor tractography and transcranial magnetic stimulation follow-up study. J Comput Assist Tomogr 2008;32:418-420.
12. Konishi J, Yamada K, Kizu O, Ito H, Sugimura K, Yoshikawa K, et al. MR tractography for the evaluation of functional recovery from lenticulostriate infarcts. Neurology 2005;64:108-113.

13. Jayaram G, Stagg CJ, Esser P, Kischka U, Stinear J, JohansenBerg $\mathrm{H}$. Relationships between functional and structural corticospinal tract integrity and walking post stroke. Clin Neurophysiol 2012;123:2422-2428.

14. Palmer JA, Needle AR, Pohlig RT, Binder-Macleod SA. Atypical cortical drive during activation of the paretic and nonparetic tibialis anterior is related to gait deficits in chronic stroke. Clin Neurophysiol 2016;127:716-723.

15. Hendricks HT, Pasman JW, van Limbeek J, Zwarts MJ. Motor evoked potentials of the lower extremity in predicting motor recovery and ambulation after stroke: a cohort study. Arch Phys Med Rehabil 2003;84:1373-1379.

16. Piron L, Piccione F, Tonin P, Dam M. Clinical correlation between motor evoked potentials and gait recovery in poststroke patients. Arch Phys Med Rehabil 2005;86:1874-1878.

17. Kwon YH, Son SM, Lee J, Bai DS, Jang SH. Combined study of transcranial magnetic stimulation and diffusion tensor tractography for prediction of motor outcome in patients with corona radiata infarct. $J$ Rehabil Med 2011;43:430-434.

18. Jang SH, Ahn SH, Sakong J, Byun WM, Choi BY, Chang CH, et al. Comparison of TMS and DT for predicting motor outcome in intracerebral hemorrhage. J Neurol Sci 2010;15:107-111.

19. Fugl-Meyer AR, Jääskö L, Leyman I, Olsson S, Steglind S. The post-stroke hemiplegic patient. 1. a method for evaluation of physical performance. Scand J Rehabil Med 1975;7:13-31.

20. Holden MK, Gill KM, Magliozzi MR, Nathan J, Piehl-Baker L. Clinical gait assessment in the neurologically impaired. Reliability and meaningfulness. Phys Ther 1984;64:35-40.

21. Jung HY, Park BK, Shin HS, Kang YK, Pyun SB, Paik NJ, et al. Development of the Korean Version of Modified Barthel Index (K-MBI): multi-center study for subjects with stroke. J Korean Acad Rehabil Med 2007;31:283-297.

22. Holden MK, Gill KM, Magliozzi MR. Gait assessment for neurologically impaired patients. Standards for outcome assessment. Phys Ther 1986;66:1530-1539.

23. Rossini PM, Barker AT, Berardelli A, Caramia MD, Caruso G, Cracco RQ, et al. Non-invasive electrical and magnetic stimulation of the brain, spinal cord and roots: basic principles and procedures for routine clinical application. Report of an IFCN committee. Electroencephalogr Clin Neurophysiol 1994;91:7992.

24. Ross ED. Localization of the pyramidal tract in the internal capsule by whole brain dissection. Neurology 1980;30:59-64.

25. Rorden C, Brett M. Stereotaxic display of brain lesions. Behav Neurol 2000;12:191-200. 
26. Lee DH, Lee DW, Han BS. Topographic organization of motor fibre tracts in the human brain: findings in multiple locations using magnetic resonance diffusion tensor tractography. Eur Radiol 2016;26:1751-1759

27. Biller J, Love BB, Marsh EE 3rd, Jones MP, Knepper LE, Jiang $D_{\text {, }}$ et al. Spontaneous improvement after acute ischemic stroke. $A$ pilot study. Stroke 1990;21:1008-1012.

28. Olsen TS. Arm and leg paresis as outcome predictors in stroke rehabilitation. Stroke 1990;21:247-251.

29. Wade DT, Langton-Hewer R, Wood VA, Skilbeck CE, Ismail HM. The hemiplegic arm after stroke: measurement and recovery. $J$ Neurol Neurosurg Psychiatry 1983;46:521-524.

30. Nakayama $H$, Jørgensen HS, Raaschou HO, Olsen TS. Recovery of upper extremity function in stroke patients: the Copenhagen Stroke Study. Arch Phys Med Rehabil 1994;75:394-398.

31. Brott $T$, Adams HP Jr, Olinger CP, Marler JR, Barsan WG, Biller $J$, et al. Measurements of acute cerebral infarction: a clinical examination scale. Stroke 1989;20:864-870.

32. Baird AE, Dambrosia J, Janket $S$, Eichbaum $Q$, Chaves $C$, Silver $B$, et al. A three-item scale for the early prediction of stroke recovery. Lancet 2001;357:2095-2099.

33. Saunders DE, Clifton AG, Brown MM. Measurement of infarct size using MRI predicts prognosis in middle cerebral artery infarction. Stroke 1995;26:2272-2276.

34. Barber PA, Demchuk AM, Zhang J, Buchan AM. Validity and reliability of a quantitative computed tomography score in predicting outcome of hyperacute stroke before thrombolytic therapy. ASPECTS Study Group. Alberta Stroke Programme Early CT Score. Lancet 2000;355:1670-1674.

35. Hübers A, Klein JC, Kang JS, Hilker R, Ziemann U. The relationship between TMS measures of functional properties and DTI measures of microstructure of the corticospinal tract. Brain Stimul 2012;5:297-304.

36. Lai C, Zhang SZ, Liu HM, Zhou YB, Zhang YY, Zhang OW, et al. White matter tractography by diffusion tensor imaging plays an important role in prognosis estimation of acute lacunar infarctions. Br J Radiol 2007;80:782-789.

37. Jang SH, Choi BY, Chang $\mathrm{CH}$, Kim SH, Chang MC. Prediction of motor outcome based on diffusion tensor tractography findings in thalamic hemorrhage. Int J Neurosci 2013;123:233239.

38. Fink JN, Selim MH, Kumar S, Silver B, Linfante I, Caplan LR, et al. Is the association of National Institutes of Health Stroke Scale scores and acute magnetic resonance imaging stroke volume equal for patients with right- and left-hemisphere ischemic stroke? Stroke 2002;33:954-958.

39. Johnston KC, Wagner DP, Haley EC Jr, Connors AF Jr; RANTTAS Investigators. Randomized trial of tirilazad mesylate in acute stroke. Combined clinical and imaging information as an early stroke outcome measure. Stroke 2002;33:466-472.

40. Wöhrle JC, Behrens S, Mielke O, Hennerici MG. Early motor evoked potentials in acute stroke: adjunctive measure to MRI for assessment of prognosis in acute stroke within 6 hours. Cerebrovasc Dis 2004;18:130-134. 